\title{
ON CONJUGATE POINTS AND GEODESIC LOOPS IN A COMPLETE RIEMANNIAN MANIFOLD
}

\author{
SHICHENG XU
}

\begin{abstract}
A well-known Lemma in Riemannian geometry by Klingenberg says that if $x_{0}$ is a minimum point of the distance function $d(p, \cdot)$ to $p$ in the cut locus $C_{p}$ of $p$, then either there is a minimal geodesic from $p$ to $x_{0}$ along which they are conjugate, or there is a geodesic loop at $p$ that smoothly goes through $x_{0}$. In this paper, we prove that: for any point $q$ and any local minimum point $x_{0}$ of $F_{q}(\cdot)=d(p, \cdot)+d(q, \cdot)$ in $C_{p}$, either $x_{0}$ is conjugate to $p$ along each minimal geodesic connecting them, or there is a geodesic from $p$ to $q$ passing through $x_{0}$. In particular, for any local minimum point $x_{0}$ of $d(p, \cdot)$ in $C_{p}$, either $p$ and $x_{0}$ are conjugate along every minimal geodesic from $p$ to $x_{0}$, or there is a geodesic loop at $p$ that smoothly goes through $x_{0}$. Earlier results based on injective radius estimate would hold under weaker conditions.
\end{abstract}

\section{INTRODUCTION}

Let $M$ be a complete Riemannian manifold. For a point $p \in M$, let $T_{p} M$ be the tangent space at $p$ and $\exp _{p}: T_{p} M \rightarrow M$ be the exponential map. For any unit vector $v \in T_{p} M$, let $\sigma(v)$ be the supremum of $l$ such that the geodesic $\exp t v:[0, l] \rightarrow M$ is minimizing, $\kappa(v)$ be the supremum of $s$ such that there is no conjugate point of $p$ along $\exp _{p} t v:[0, s) \rightarrow M$. Let

$$
\tilde{C}_{p}=\left\{\sigma(v) v: \text { for all unit vector } v \in T_{p} M\right\}
$$

be the tangential cut locus of $p$,

$$
\tilde{J}_{p}=\left\{\kappa(v) v: \text { for all unit vector } v \in T_{p} M\right\}
$$

be the tangential conjugate locus, and $C_{p}=\exp \tilde{C}_{p} \subset M$ be the cut locus of $p$. Let

$$
\tilde{D}_{p}=\left\{t v \mid 0 \leq t<\sigma(v) \text {, for all unit vector } v \in T_{p} M\right\}
$$

be the maximal open domain of the origin in $T_{p} M$ such that the restriction $\left.\exp _{p}\right|_{\tilde{D}_{p}}$ of $\exp _{p}$ on $\tilde{D}_{p}$ is injective. Any geodesic throughout the paper is assumed to be parametrized by arclength. A geodesic $\gamma:[0, l] \rightarrow M$ is called a geodesic loop at $p$ if $\gamma(0)=\gamma(l)=p$. Let $d(\cdot, \cdot)$ be the Riemannian distance function on $M$. In [8] Klingenberg proved a lemma that is wellknown now in Riemannian geometry and particularly useful in injectivity radius estimate.

Date: January 24, 2014.

Keywords: geodesic, cut point, conjugate point, injectivity radius.

2000 MSC: Primary 53C22; Secondary 53C20.

Project 11171143 supported by National Natural Science Foundation of China. 
Lemma 1.1 (Klingenberg $[8,9])$. If $x_{0} \in C_{p}$ satisfies that $d\left(p, x_{0}\right)=$ $d\left(p, C_{p}\right)$, then either there is a minimal geodesic from $p$ to $x_{0}$ along which they are conjugate, or there exist exactly two minimal geodesics from $p$ to $x_{0}$ that form a geodesic loop at $p$ smoothly passing through $x_{0}$.

Recently this lemma was generalized to the case of two points ([7]). Let $p, q$ be two points in a complete Riemannian manifold $M$ such that $C_{p} \neq \emptyset$ and $q \notin C_{p}$. Let $F_{p ; q}: C_{p} \rightarrow \mathbb{R}$ be a function defined on $C_{p}$ by $F_{p ; q}(x)=$ $d(p, x)+d(x, q)$. Innami, Shiohama and Soga proved in [7] that

Lemma 1.2 ([7]). If $\tilde{J}_{p} \cap \tilde{C}_{p}=\emptyset$, then for any minimum point $x_{0} \in C_{p}$ of $F_{p ; q}(x)=d(p, x)+d(x, q)$, there exist a geodesic (and at most two) $\alpha$ : $\left[0, F_{p ; q}\left(x_{0}\right)\right] \rightarrow M$ from $p$ to $q$ such that $\alpha\left(d\left(p, x_{0}\right)\right)=x_{0}$.

Note that if $p=q$, then Lemma 1.2 is reduced to the case of Klingenberg's Lemma. In this paper, we improve both results in the above to the following theorems whose constraints are sharp in general.

Theorem A (Generalized Klingenberg's Lemma). Let $M$ be a complete Riemannian manifold and $p, q \in M$ such that $C_{p} \neq \emptyset$ and $q \notin C_{p}$. Let $x_{0} \in$ $C_{p}$ such that $F_{p ; q}\left(x_{0}\right)=d\left(p, x_{0}\right)+d\left(q, x_{0}\right)$ is local minimum of $F_{p ; q}$ in $C_{p}$. Then either $p$ and $x_{0}$ are conjugate along every minimal geodesic connecting them, or there is a geodesic (and at most two) $\alpha:\left[0, F_{p ; q}\left(x_{0}\right)\right] \rightarrow M$ from $p$ to $q$ such that $\alpha\left(d\left(p, x_{0}\right)\right)=x_{0}$.

Theorem B (Improved Klingenberg's Lemma). Let $M$ be a Riemannian manifold and $p$ be a point in $M$. Let $x_{0} \in C_{p}$ such that $d\left(p, x_{0}\right)$ is a local minimum of $d(p, \cdot)$ in $C_{p}$. If there is a minimal geodesic from $p$ to $x_{0}$ along which $p$ is not conjugate to $x_{0}$, then there are exactly two minimal geodesics from $p$ to $x_{0}$ that form a whole geodesic smoothly passing through $x_{0}$.

Moreover, if $d\left(p, x_{0}\right)$ is also a local minimum of $d\left(x_{0}, \cdot\right)$ in $C_{x_{0}}$, then the two minimal geodesics form a closed geodesic.

Motivated by Theorem B, we will call a cut point $q$ of $p$ an essential conjugate point of $p$ if $p$ is conjugate to $q$ along every minimal geodesic connecting them. Geodesic loops may not exist if there are essential conjugate points. For example, a complete noncompact Riemannian manifold of positive sectional curvature always contains a simple point $p$ (i.e. there is no geodesic loop at $p$ ) whose cut locus is nonempty [6]. By Theorem B, every local minimum point of $d(p, \cdot)$ in $C_{p}$ must be essentially conjugate to $p$.

The conjugate radius had been involved in the injectivity radius estimate besides the length of geodesic loops (see [8, Lemma 4] or [1, Lemma 1.8]). Recall that the conjugate radius at $p$ is defined by

$$
\operatorname{conj}(p)=\min \left\{\kappa(v) \mid \text { for all unit vector } v \in T_{p} M\right\}
$$

and the conjugate radius of $M, \operatorname{conj}(M)=\inf _{p \in M} \operatorname{conj}(p)$. The injectivity radius of $p$ is defined by

$$
\operatorname{injrad}(p)=\min \left\{\sigma(v) \mid \text { for all unit vector } v \in T_{p} M\right\}
$$

and injectivity radius of $M, \operatorname{injrad}(M)=\inf _{p \in M} \operatorname{injrad}(p)$. It follows from Theorem $\mathrm{B}$ that the conjugate radius in the injectivity radius estimate can be replaced by the distance from $p$ to its essential conjugate points. Let 
$\left.J_{p}^{\varepsilon}=C_{p} \backslash \exp _{p}\left(\tilde{C}_{p} \backslash \tilde{J}_{p}\right)\right)$ be the the set consisting of all essential conjugate points of $p$. Let

$$
\operatorname{conj}^{\varepsilon}(p)= \begin{cases}d\left(p, J_{p}^{\varepsilon}\right) & \text { if } J_{p}^{\varepsilon} \neq \emptyset, \\ +\infty & \text { otherwise. }\end{cases}
$$

and $\operatorname{conj}^{\varepsilon}(M)=\min _{p \in M} \operatorname{conj}^{\varepsilon}(p)$. Then the injectivity radius can be expressed in the following way, where non-essential conjugate points are covered by geodesic loops.

\section{Theorem 1.3.}

$$
\begin{aligned}
& \operatorname{injrad}(p)=\min \left\{\begin{array}{l}
\operatorname{conj}^{\varepsilon}(p), \\
\text { half length of the shortest geodesic loop at } p
\end{array}\right\} ; \\
& \operatorname{injrad}(M)=\min \left\{\begin{array}{l}
\operatorname{conj}^{\varepsilon}(M), \\
\text { half length of the shortest closed geodesic in } M
\end{array}\right\} .
\end{aligned}
$$

By Theorem 1.3, in this paper we call $\operatorname{conj}^{\varepsilon}(p)$ the essential conjugate radius of $p$ and $\operatorname{conj}^{\varepsilon}(M)$ the essential conjugate radius of $M$.

There have been rich results in Riemannian geometry where an upper sectional curvature bound $K$ shows up to offer a lower bound $\frac{\pi}{\sqrt{K}}$ of the conjugate radius, which now could be weakened to the essential conjugate radius. For instance, one is able to generalize Cheeger's finiteness theorem of diffeomorphism classes to Riemannian manifolds whose essential conjugate radius has a lower bound but sectional curvature has no upper bound.

Theorem 1.4. For any positive integer $n$, real numbers $D, v, r>0$ and $k$, there are only finite $C^{\infty}$-diffeomorphism classes in the set consisting of $n$-dimensional Riemannian manifolds whose sectional curvature is bounded below by $k$, diameter $\leq D$, volume $\geq v$ and essential conjugate radius $\geq r$.

In general $\operatorname{conj}^{\varepsilon}(p) \neq \operatorname{conj}(p) . \mathbb{R} P^{n}$ with the canonical metric is a trivial example. An immediate question is, when $\operatorname{conj}^{\varepsilon}(p)=\operatorname{conj}(p)$ ? Next two applications of Theorem B offers examples where $\operatorname{conj}^{\varepsilon}(p)$ coincides with $\operatorname{conj}(p)$.

Theorem 1.5. Let a point $p$ be the soul of a complete noncompact Riemannian manifold of nonnegative sectional curvature in sense of [4]. Then either the nearest point to $p$ in $C_{p}$ is an essential conjugate point, or $\exp _{p}$ : $T_{p} M \rightarrow M$ is a diffeomorphism.

By Theorem B, Theorem 1.5 directly follows from the fact that the soul is totally convex $([4])$. For a closed Riemannian manifold $M$, the radius of $M$ is defined by $\operatorname{rad}(M)=\min _{p \in M} \max \{d(p, x) \mid$ for any $x \in M\}$.

Theorem 1.6. Let $M$ be a complete Riemannian manifold whose sectional curvature $\geq 1$ and radius $\operatorname{rad}(M)>\frac{\pi}{2}$. Then either there are two points $p, q \in M$ of distance $d(p, q)=d\left(p, C_{p}\right)=d\left(q, C_{q}\right)<\operatorname{rad}(M)$ and $p, q$ are essentially conjugate to each other, or $M$ is isometric to a sphere of constant curvature $\frac{\pi^{2}}{\operatorname{rad}^{2}(M)}$.

Xia proved ([11]) that if the manifold $M$ in Theorem 1.6 satisfies $\operatorname{conj}(M) \geq$ $\operatorname{rad}(M)>\frac{\pi}{2}$, then $M$ is isometric to a sphere of constant curvature. Theorem 1.6 follows his proof after replacing Klingenberg's lemma to Theorem B. 
In particular, if $\operatorname{rad}(p)=\max _{x \in M} d(p, x)>\frac{\pi}{2}$, then $\operatorname{injrad}(p)=\operatorname{conj}^{\varepsilon}(p)=$ $\operatorname{conj}(p)$. Weaker versions of Theorem 1.6 can also be found in [10].

Theorem A provides a new characterization on general Riemannian manifolds, which is an improvement of Theorem 1 in [7].

Theorem 1.7. Let $M$ be a complete Riemannian manifold and $p$ be a point in $M$ such that $C_{p} \neq \emptyset$. Then

(1.7.1) either $p$ has an essential conjugate point;

(1.7.2) or there exist at least two geodesics connecting $p$ and every point $q \in M$ (regarding the single point $p$ as a geodesic when $p=q$ ).

We now explain the idea of our proof of Theorem A. Let $p, q \in M$ such that $C_{p} \neq \emptyset, q \notin C_{p}$ and $x_{0} \in C_{p}$ is a minimum point of $F_{p ; q}$ in $C_{p}$. Let us consider the special case that there is a unique minimal geodesic $\left[q x_{0}\right]$ connecting $q$ and $x_{0}$. A key observation from [7] is that the level set of $\left\{F_{p ; q} \leq C\right\}$ is star-shaped at both $p$ and $q$. In particular, if two minimal geodesics $\left[p x_{0}\right]$ and $\left[x_{0} q\right]$ from $p$ to $x_{0}$ and from $x_{0}$ to $q$ are broken at $x_{0}$, then for any point $x \neq x_{0}$ in $\left[p x_{0}\right]$ and any minimal geodesic $[q x]$ connecting $q$ and $x$, we have $[q x] \cap C_{p}=\emptyset$. Thus $[q x]$ admit a unique lifting $\widetilde{[q x]}$ in $\tilde{D}_{p}$. If two minimal geodesics, say $\left[p x_{0}\right]_{1}$ and $\left[p x_{0}\right]_{2}$, do not form a whole geodesic with $\left[x_{0} q\right]$ at the same time, then by moving $x$ to $x_{0}$ along $\left[p x_{0}\right]_{1}$ and $\left[p x_{0}\right]_{2}$ respectively, one may expect two liftings of $\left[q x_{0}\right]$ in $\tilde{D}_{p}$ with different endpoints when partial limits of $\widetilde{[q x]}$ exist.

Under the condition that $\tilde{J}_{p} \cap \tilde{C}_{p}=\emptyset$ as in Lemma 1.2 and [7], all such minimal geodesics $[p x]$ are clearly definite away from the tangential conjugate locus. So we are able to take limit to meet a contradiction, for the lift $\widetilde{\left[q x_{0}\right]}$ of $\left[q x_{0}\right]$ is unique in the closure of $\tilde{D}_{p}$.

In the general case one does not know whether $\left[q x_{0}\right]$ admits a lifting at its endpoint in the closure of $\tilde{D}_{p}$, nor the liftings $\widetilde{[q x]}$ have a partial limit as $x$ approaches $x_{0}$ along $\left[p x_{0}\right]$. We will prove that, if there is a minimal geodesic $\alpha$ from $p$ to $x_{0}$ along which they are not conjugate to each other, and the union of $\alpha$ and $\left[q x_{0}\right]$ is broken at $x_{0}$, then $\left[q x_{0}\right]$ always has a lifting in the closure of $\tilde{D}_{p}$, which share a common endpoint with $\tilde{\alpha}$ (see Lemma 2.2).

The uniqueness of the minimal geodesic $\left[q x_{0}\right]$ is not an essential problem, because by (2.2.4) and (2.2.5) we are always able to move $q$ along $\left[q x_{0}\right]$ while keeping $F_{p ; q}$ minimal at $x_{0}$ in $C_{p}$. A local minimum of $F_{p ; q}$ can be reduced to the minimum case similarly.

The detailed proof of Theorem A will be given in the next section.

\section{Proof of the generalized Klingenberg's lemma}

In this section we will prove Theorem A. Let $M$ be a complete Riemannian manifold and $p, q$ be two points in $M$ such that $C_{p} \neq \emptyset$ and $q \notin C_{p}$. Let us consider the function

$$
F_{p ; q}: C_{p} \rightarrow \mathbb{R}, \quad F_{p ; q}(x)=d(x, q)+d(x, p)
$$

and assume that $F_{p ; q}$ takes its minimum at $x_{0} \in C_{p}$. Because $q$ is not a cut point of $p, q \neq x_{0}$. Let $\gamma:\left[0, d\left(q, x_{0}\right)\right] \rightarrow M$ be a minimal geodesic 
from $q=\gamma(0)$ to $x_{0}=\gamma\left(d\left(q, x_{0}\right)\right)$. Because $x_{0}$ is a cut point of $p$, for any $0 \leq t<d\left(q, x_{0}\right)$ we have

$$
\begin{aligned}
F_{q}(\gamma(t)) & =d(q, \gamma(t))+d(\gamma(t), p) \\
& <d(q, \gamma(t))+d\left(\gamma(t), x_{0}\right)+d\left(x_{0}, p\right) \\
& =d\left(q, x_{0}\right)+d\left(x_{0}, p\right) \\
& =F_{q}\left(x_{0}\right) \\
& =\min F_{q} .
\end{aligned}
$$

Therefore $\gamma(t)\left(0 \leq t<d\left(q, x_{0}\right)\right)$ is not a cut point of $p$, and we are able to lift $\left.\gamma\right|_{\left[0, d\left(q, x_{0}\right)\right)}$ to $\left(\left.\exp _{p}\right|_{\tilde{D}_{p}}\right)^{-1} \circ \gamma$ uniquely in the tangential segment domain $\tilde{D}_{p} \subset T_{p} M$, where $\tilde{D}_{p}$ is the maximal open domain of the origin in $T_{p} M$ such that the restriction $\left.\exp _{p}\right|_{\tilde{D}_{p}}$ of $\exp _{p}$ on $\tilde{D}_{p}$ is injective.

We now prove that, if $x_{0} \in \exp _{p}(\tilde{C}(p) \backslash \tilde{J}(p))$, then either $\gamma$ can be lifted on the whole interval $\left[0, d\left(q, x_{0}\right)\right]$ such that the endpoint of the lift is a regular point of $\exp _{p}$, or $\gamma$ can be extended to a geodesic from $q$ to $p$ that goes through $x_{0}$.

Lemma 2.2. Assume that there is a minimal geodesic $\alpha:\left[0, d\left(p, x_{0}\right)\right] \rightarrow M$ from $p=\alpha(0)$ to $x_{0}=\alpha\left(d\left(p, x_{0}\right)\right)$ along which $p$ is not conjugate to $x_{0}$. Let $w=d\left(p, x_{0}\right) \alpha^{\prime}(0) \in T_{p} M$, where $\alpha^{\prime}(0)$ is the unit tangent vector of $\alpha$ at $p$. Then

(2.2.1) either $\gamma$ and $\alpha$ form a whole geodesic at $x_{0}$;

(2.2.2) or there is a unique smooth lift $\tilde{\gamma}:\left[0, d\left(q, x_{0}\right)\right] \rightarrow T_{p} M$ of $\gamma:$ $\left[0, d\left(q, x_{0}\right)\right] \rightarrow M$ in the tangential segment domain $\tilde{D}_{p} \subset T_{p} M$ such that $\tilde{\gamma}(0)=\left(\left.\exp _{p}\right|_{\tilde{D}_{p}}\right)^{-1} \gamma(0)$ and $\tilde{\gamma}\left(d\left(q, x_{0}\right)\right)=w$.

Proof. We first prove the case that $\gamma$ is the unique minimal geodesic from $q$ to $x_{0}$. Assume that $\gamma$ and $\alpha$ do not form a whole geodesic at $x_{0}$. Let $\left\{\alpha\left(s_{i}\right)\right\}$ $\left(0<s_{i}<d\left(p, x_{0}\right)\right)$ be a sequence of interior points of $\alpha$ that converges to $x_{0}$ as $i \rightarrow \infty$, and let $\gamma_{s_{i}}:\left[0, d\left(q, \alpha\left(s_{i}\right)\right)\right] \rightarrow M$ be a minimal geodesic from $q$ to $\alpha\left(s_{i}\right)$. Then $\gamma_{s_{i}}$ converges to $\gamma$ as $i \rightarrow \infty$.

Because $\gamma$ and $\alpha$ are broken at $x_{0}$, by the triangle inequality and similar calculation in $(2.1)$, for each $s_{i}$, we have

$$
F_{p ; q}\left(\alpha\left(s_{i}\right)\right)<F_{p ; q}\left(x_{0}\right)=\min F_{p ; q},
$$

and thus for any $0 \leq t \leq d\left(q, \alpha\left(s_{i}\right)\right)$,

$$
F_{p ; q}\left(\gamma_{s_{i}}(t)\right) \leq F_{p ; q}\left(\alpha\left(s_{i}\right)\right)<\min F_{p ; q},
$$

which implies that none of points in $\gamma_{s_{i}}$ is a cut point of $p$. Therefore there is a unique lift curve $\tilde{\gamma}_{s_{i}}$ of $\gamma_{s_{i}}$ starting at $\left(\exp _{p} \mid \tilde{D}_{p}\right)^{-1} \gamma(0)$ in the tangential segment domain $\tilde{D}_{p} \subset T_{p} M$.

If $\left\{\tilde{\gamma}_{s_{i}}\right\}$ is uniformly Lipschitz, then by the Arzelà-Ascoli theorem, a subsequence of $\left\{\tilde{\gamma}_{s_{i}}\right\}$ converges to a continuous curve $\tilde{\gamma}_{\infty}:\left[0, d\left(q, x_{0}\right)\right] \rightarrow T_{p} M$, which satisfies that

and

$$
\tilde{\gamma}_{\infty}(0)=\left(\left.\exp _{p}\right|_{\tilde{D}_{p}}\right)^{-1} \gamma(0), \quad \tilde{\gamma}_{\infty}\left(d\left(q, x_{0}\right)\right)=w,
$$

$$
\exp _{p}\left(\tilde{\gamma}_{\infty}(t)\right)=\lim _{i \rightarrow \infty} \gamma_{s_{i}}(t)=\gamma(t), \text { for all } 0 \leq t \leq d\left(q, x_{0}\right)
$$


That is, $\gamma$ has a unique lifting in the segment domain that satisfies (2.2.2).

To prove that $\left\{\tilde{\gamma}_{s_{i}}\right\}$ is uniformly Lipschitz, it suffices to show that there is $N>0$ such that the distance between $\bigcup_{i \geq N} \tilde{\gamma}_{s_{i}}\left(\left[0, d\left(q, \alpha\left(s_{i}\right)\right)\right]\right)$ and tangential conjugate locus $\tilde{J}_{p} \subset T_{p} M$ is positive. Indeed, because $\tilde{\gamma}_{s_{i}}\left(d\left(q, \alpha\left(s_{i}\right)\right)\right)$ converges to $w=d\left(p, x_{0}\right) \alpha^{\prime}(0)$, at which the differential $d\left(\exp _{p}\right)$ is nonsingular, there is a small $\delta>0$ and some $\epsilon>0$ such that

$$
d\left(\tilde{\gamma}_{s_{i}}(t), \tilde{J}_{p}\right)>\epsilon \text {, for all } d\left(q, \alpha\left(s_{i}\right)\right)-\delta \leq t \leq d\left(q, \alpha\left(s_{i}\right)\right) \text { and large } i \text {. }
$$

On the other hand, because the restriction $\left.\gamma_{s_{i}}\right|_{\left[0, d\left(q, \alpha\left(s_{i}\right)\right)-\delta\right]}$ converges to $\left.\gamma\right|_{\left[0, d\left(q, x_{0}\right)-\delta\right]}$, which lies in the segment domain $D_{p}=\exp _{p} \tilde{D}_{p} \subset M$, there is some $\epsilon_{1}>0$ such that

$$
d\left(\tilde{\gamma}_{s_{i}}(t), \tilde{J}_{p}\right)>\epsilon_{1}, \text { for all } 0 \leq t \leq d\left(q, \alpha\left(s_{i}\right)\right)-\delta \text { and large } i .
$$

Now what remains is to prove the case that the minimal geodesic from $q$ to $x_{0}$ is not unique. Let us fix some interior point $q_{1}=\gamma\left(t_{1}\right)\left(0<t_{1}<d\left(q, x_{0}\right)\right)$ of $\gamma$ and consider the function $F_{p ; q_{1}}: C_{p} \rightarrow \mathbb{R}$ instead. Because

$$
\begin{aligned}
F_{p ; q_{1}}(x) & =d\left(q_{1}, x\right)+d(x, p) \\
& \geq d(x, q)-d\left(q, q_{1}\right)+d(x, p) \\
& =F_{p ; q}(x)-d\left(q, q_{1}\right)
\end{aligned}
$$

and

$$
\begin{aligned}
F_{p ; q_{1}}\left(x_{0}\right) & =d\left(x_{0}, q\right)-d\left(q, q_{1}\right)+d\left(x_{0}, p\right) \\
& =F_{p ; q}\left(x_{0}\right)-d\left(q, q_{1}\right),
\end{aligned}
$$

we see that $F_{p ; q_{1}}(x)$ also takes minimum at $x_{0}$. Now the minimal geodesic from $q_{1}$ to $x_{0}$ is unique. By the same argument as above, either $\left.\gamma\right|_{\left[t_{1}, d\left(q, x_{0}\right)\right]}$ from a whole geodesic with $\alpha$ at $x_{0}$, or it has a unique lift in the tangential segment domain. So does $\gamma$.

A local minimum point of $F_{p ; q}$ can be reduced to the case of global minimum by the following lemma.

Lemma 2.3. Let $x_{0}$ be a local minimum point of $F_{p ; q}: C_{p} \rightarrow \mathbb{R}$ in $C_{p}$, and $\gamma:\left[0, d\left(q, x_{0}\right)\right] \rightarrow M$ be a minimal geodesic from $q=\gamma(0)$ to $x_{0}=$ $\gamma\left(d\left(q, x_{0}\right)\right)$. Then for any interior point $q_{t}=\gamma(t)$ of $\gamma$ that is sufficient close to $x_{0}$, the function $F_{p ; q_{t}}: C_{p} \rightarrow \mathbb{R}$ takes its minimum at $x_{0}$.

Proof. By (2.2.4) and (2.2.5), $x_{0}$ is also a local minimum point of $F_{p ; q_{t}}$ for any $t \in\left[0, d\left(q, x_{0}\right)\right)$. Therefore, it suffices to show that $F_{p ; q_{t}}$ takes its minimum near $x_{0}$ as $q_{t}$ sufficient close to $x_{0}$.

Let us argue by contradiction. Assuming the contrary, one is able to find a sequence of points $\left\{q_{i}=\gamma\left(t_{i}\right)\right\}$ that converges to $x_{0}$ such that $F_{p ; q_{i}}$ takes its minimum at some point $z_{i} \in C_{p}$ outside an open ball of $x_{0}$,

$$
B_{\epsilon}\left(x_{0}\right)=\left\{x \in M \mid d\left(x, x_{0}\right)<\epsilon\right\} .
$$

By passing to a subsequence, we assume that $z_{i} \rightarrow z_{0} \in C_{p}$. Then for any $y \in C_{p}$,

$$
d\left(p, z_{i}\right)+d\left(z_{i}, q_{i}\right)=F_{p ; q_{i}}\left(z_{i}\right) \leq F_{p ; q_{i}}(y)=d(p, y)+d\left(y, q_{i}\right) .
$$


Taking limit of the above inequality, we get

$$
F_{p ; x_{0}}\left(z_{0}\right) \leq F_{p ; x_{0}}(y), \quad \text { for any } y \in C_{p} .
$$

Let $y=x_{0}$, then

$$
d\left(p, z_{0}\right)+d\left(z_{0}, x_{0}\right) \leq F_{p ; x_{0}}\left(x_{0}\right)=d\left(p, x_{0}\right) .
$$

Because $d\left(z_{0}, x_{0}\right) \geq \epsilon$, this implies that $z_{0}$ is an interior point of a minimal geodesic from $p$ to $x_{0}$, and it contradicts to the fact that $z_{0} \in C_{p}$.

We now are ready to prove Theorem A.

Proof of Theorem A. Let $x_{0} \in C_{p}$ be a local minimum point of the function

$$
F_{p ; q}: C_{p} \rightarrow \mathbb{R}, \quad F_{p ; q}(x)=d(x, q)+d(x, p) .
$$

Let $\alpha:\left[0, d\left(p, x_{0}\right)\right] \rightarrow M$ be a minimal geodesic from $p=\alpha(0)$ to $x_{0}=$ $\alpha\left(d\left(p, x_{0}\right)\right)$, along which $p$ is not conjugate to $x_{0}$. Let $\gamma:\left[0, d\left(q, x_{0}\right)\right] \rightarrow M$ be a minimal geodesic from $q=\gamma(0)$ to $x_{0}=\gamma\left(d\left(q, x_{0}\right)\right)$, and $w=d\left(p, x_{0}\right) \alpha^{\prime}(0)$.

First let us prove that there is a minimal geodesic from $p$ to $x_{0}$ that forms a whole geodesic with $\gamma$. According to Lemma 2.3, by moving $q$ to an interior point in $\gamma$ that is sufficient close to $x_{0}$ and denoted also by $q$, it can be reduced to the case that $x_{0}$ is a minimal point of $F_{p ; q}$ and $\gamma$ is a unique minimal geodesic connecting $q$ and $x_{0}$.

By Lemma 2.2, if $\alpha$ and $\gamma$ are broken at $x_{0}$, then $\gamma$ has a unique lift $\tilde{\gamma}:\left[0, d\left(q, x_{0}\right)\right] \rightarrow T_{p} M$ in the tangential segment domain $\tilde{D}_{p} \subset T_{p} M$, whose endpoint satisfies

$$
\tilde{\gamma}\left(d\left(q, x_{0}\right)\right)=w
$$

Because $x_{0}$ is not conjugate to $p$ along $\alpha$, there is another minimal geodesic $\beta:\left[0, d\left(p, x_{0}\right)\right] \rightarrow M$ from $p$ to $x_{0}$. We now prove that $\beta$ must form a whole geodesic with $\gamma$.

Assuming the contrary, that is, $\beta$ does not form a whole geodesic with $\gamma$ neither. For any interior point $\beta(s)\left(0<s<d\left(p, x_{0}\right)\right)$ in $\beta$, let $l_{s}=d(q, \beta(s))$ and $\gamma_{s}:\left[0, l_{s}\right] \rightarrow M$ be a minimal geodesic from $q$ to $\beta(s)$. Then by the same argument as (2.2.3), for any $0 \leq t \leq l_{s}$ we have

$$
F_{p ; q}\left(\gamma_{s}(t)\right) \leq F_{p ; q}(\beta(s))<F_{p ; q}\left(x_{0}\right)=\min F_{p ; q},
$$

which implies that $\gamma_{s}$ has a unique lift $\tilde{\gamma}_{s}$ in the tangential segment domain $\tilde{D}_{p} \subset T_{p} M$.

We point it out that, because the endpoint $\tilde{\gamma}\left(l_{s}\right)$ of $\tilde{\gamma}_{s}$ may approach $\tilde{J}_{p} \subset T_{p} M$, one cannot directly conclude that the family of curves $\tilde{\gamma}_{s}$ contains any convergent subsequence as $s \rightarrow d\left(p, x_{0}\right)$. Instead, let us consider a small metric ball $B_{\epsilon}(w)$ at $w=d\left(p, x_{0}\right) \alpha^{\prime}(0)$ in $T_{p} M$ on which the restriction of $\exp _{p}$

$$
\left.\exp _{p}\right|_{B_{\epsilon}(w)}: B_{\epsilon}(w) \rightarrow M
$$

is an diffeomorphism onto its image. Then $d\left(p, x_{0}\right) \beta^{\prime}(0) \notin B_{\epsilon}(w)$. Because $\gamma_{s}$ converges to $\gamma$ as $s \rightarrow d\left(p, x_{0}\right)$ and the restriction $\left.\exp _{p}\right|_{\tilde{D}_{p}}$ of $\exp _{p}$ on the tangential segment domain $\tilde{D}_{p}$ is a diffeomorphism, $\left.\tilde{\gamma}_{s}\right|_{\left[0, l_{s}\right)}$ converges to $\left.\tilde{\gamma}_{s}\right|_{\left[0, d\left(q, x_{0}\right)\right)}$ pointwisely, that is, for $0<t<1$,

$$
\tilde{\gamma}_{s}\left(l_{s} \cdot t\right) \rightarrow \tilde{\gamma}\left(d\left(q, x_{0}\right) \cdot t\right) \text { as } s \rightarrow d\left(p, x_{0}\right) .
$$


Then for any $0<t_{1}<1$ that is sufficient close to 1 , there exists $0<s\left(t_{1}\right)<$ $d\left(p, x_{0}\right)$ such that for all $s\left(t_{1}\right)<s<d\left(p, x_{0}\right)$,

$$
\tilde{\gamma}_{s}\left(l_{s} t_{1}\right) \in B_{\frac{\epsilon}{4}}(w), \quad \tilde{\gamma}_{s}\left(l_{s}\right) \in B_{\frac{\epsilon}{2}}\left(d\left(p, x_{0}\right) \beta^{\prime}(0)\right),
$$

and $\left.\gamma_{s}\right|_{\left[l_{s} t_{1}, l_{s}\right]}$ lies in the open neighborhood $\exp _{p} B_{\frac{\epsilon}{4}}(w)$ of $x_{0}$. Because the lift of $\left.\gamma_{s}\right|_{\left[l_{s} t_{1}, l_{s}\right]}$ is unique in $\tilde{D}_{p}$ and $\tilde{\gamma}_{s}\left(l_{s} t_{1}\right) \in B_{\frac{\epsilon}{4}}(w)$, we conclude that $\left.\tilde{\gamma}_{s}\right|_{\left[l_{s} t_{1}, l_{s}\right]}$ lies in $B_{\frac{\epsilon}{4}}(w)$, which contradicts to the fact that

$$
B_{\frac{\epsilon}{4}}(w) \cap B_{\frac{\epsilon}{2}}\left(d\left(p, x_{0}\right) \beta^{\prime}(0)\right)=\emptyset .
$$

Secondly, we prove that there are at most two geodesics from $q$ to $p$ passing through $x_{0}$. Indeed, if there are two distinct minimal geodesics $\gamma_{1}$, $\gamma_{2}$ connecting $q$ and $x_{0}$, then one of them, say $\gamma_{1}$, does not form a whole geodesic with $\alpha$. By the proof above, any other minimal geodesic except $\alpha$ connecting $p$ and $x_{0}$ from a whole geodesic with $\gamma_{1}$. By Lemma 2.2, $\gamma_{2}$ form a whole geodesic with $\alpha$. Therefore, there are exactly two minimal geodesics $\alpha$ and $\beta$ from $p$ to $x_{0}$, the union of $\beta$ and $\gamma_{1}$ form a whole geodesic going through $x_{0}$, and $\alpha$ and $\gamma_{2}$ form another whole geodesic. In particular, there won't be a third geodesic from $q$ to $x_{0}$.

Theorem B and Theorem 1.7 are immediate corollaries of Theorem A. We give a proof of Theorem 1.4 to end the paper.

Proof of Theorem 1.4. Let $\mathcal{M}(n, k, D, v, r)$ be the set consisting of all complete $n$-dimensional Riemannian manifolds whose sectional curvature $\geq k$, diameter $\leq D$, volume $\geq v$ and essential conjugate radius $\geq r$. Let $M \in$ $\mathcal{M}(n, k, D, v, r)$. By Cheeger's lemma ([2], see Theorem 5.8 in [3]), there is an universal constant $c_{n}(D, v, k)>0$ depending only on $n, D, v, k$ such that every smooth closed geodesic on $M$ has length $>c_{n}(D, v, k)$. Therefore, by Theorem 1.3 the injectivity radius of $M$ is bounded below by $\min \left\{r, \frac{1}{2} c_{n}(D, v, k)\right\}$. The finiteness of diffeomorphism classes in the set $\mathcal{M}(n, k, D, v, r)$ follows from the standard argument (see $[5,12]$ for example) on the construction of a diffeomorphism between two Riemannian manifolds with small Gromov-Hausdorff distance .

\section{REFERENCES}

[1] U. Abresch and W. T. Meyer. Injectivity radius estimates and sphere theorems. Comparison geometry, 30:1-47, 1997.

[2] J. Cheeger. Finiteness theorems for riemannian manifolds. Am. J. Math., 92(1):61-74, 1970.

[3] J. Cheeger and D.G. Ebin. Comparison theorems in Riemannian geometry, volume 365. AMS Bookstore, 1975.

[4] J. Cheeger and D. Gromoll. On the structure of complete manifolds of nonnegative curvature. Ann. of Math., 96(3):413-443, 1972.

[5] K. Fukaya. Collapsing riemannian manifolds to ones of lower dimensions. J. Differential Geom., 25(1):139-156, 1987.

[6] D. Gromoll and W. Meyer. On complete open manifolds of positive curvature. Ann. of Math., 90(1):75-90, 1969.

[7] N. Innami, K. Shiohama, and T. Soga. The cut loci, conjugate loci and poles in a complete riemannian manifold. Geom. Funct. Anal., 22(5):1400-1406, 2012.

[8] W. Klingenberg. Contributions to riemannian geometry in the large. Ann. of Math., 69(3):654-666, 1959. 
[9] W. Klingenberg. Riemannian geometry. Walter de Gruyter, 1995.

[10] Q. Wang. On the geometry of positively curved manifolds with large radius. Illinois J. Math., 48(1):89-96, 012004.

[11] Changyu Xia. A round sphere theorem for positive sectional curvature. Compositio Math., 142(5):1327-1331, 2006.

[12] T. Yamaguchi. Collapsing and pinching under a lower curvature bound. Ann. of Math., 133(2):317-357, 1991.

School of Mathematics, Capital Normal University, Beijing, China

E-mail address: shichengxu@gmail.com 